\title{
GROUPS OF INVERTIBLE ELEMENTS OF BANACH ALGEBRAS ${ }^{1}$
}

\author{
BY YVONNE YUEN ${ }^{2}$
}

Communicated by Mary Ellen Rudin, July 20, 1972

\begin{abstract}
Let $A$ be a complex Banach algebra, $G$ its group of invertible elements, and $G_{e}$ the component of the identity of $G$. Then $G_{e}$ is a closed, normal subgroup of $G$. This paper contains examples of $B^{*}$ algebras $A$ for which $G / G_{e}$ is finite, but not trivial, and of a $B^{*}$ algebra for which $G / G_{e}$ is noncommutative.
\end{abstract}

Let $A$ denote a complex Banach algebra, $G$ its group of invertible elements, and $G_{e}$ the component of the identity of $G$. If $A$ is finite-dimensional, or if $A=B(H)$, the algebra of all bounded linear operators on a Hilbert space $H$, or if $A$ is commutative, then $G / G_{e}$ is torsion free. For the first two cases we actually have $G$ connected, so $G=G_{e}$. A proof of the last result, which is due to Lorch, can be found in [3, p. 15]. We shall give examples of closed, noncommutative subalgebras of $B(H)$ for which $G / G_{e}$ is finite, but not trivial, and of a $B^{*}$ algebra for which $G / G_{e}$ is not abelian. Our examples will be special cases of the following class of Banach algebras.

Let $m$ be a finite, positive Borel measure whose support is a compact Hausdorff space $X$. Let $A(X, n)$ denote the set of all continuous functions from $X$ into $M_{n}$, the algebra of all complex $n \times n$ matrices. Then $A(X, n)$ is a Banach algebra under the pointwise addition and multiplication of functions and the following norm:

$$
\|F\|=\sup _{x \in X}|F(x)|, \quad F \in A(X, n),
$$

where

$$
|F(x)|=\sup \left\{|F(x) y|: y \in C^{n}, \sum_{i=1}^{n}\left|y_{i}\right|^{2} \leqq 1\right\} .
$$

We can also define an involution on $A(X, n)$ by

$$
F^{*}(x)=(F(x))^{*} \quad \text { for } F \in A(X, n) \text { and } x \in X,
$$

where $(F(x))^{*}$ denotes the conjugate transpose of $F(x)$. Then $A(X, n)$ is a $B^{*}$-algebra under this norm and involution. For, each $F \in A(X, n)$ induces an operator $\widetilde{F}$ on $H=L^{2}(m) \oplus \cdots \oplus L^{2}(m)$ by

$$
(\tilde{F} f)(x)=F(x) f(x) \text { for } f \in H \text { and } x \in X \text {. }
$$

AMS (MOS) subject classifications (1970). Primary 46L05, 46D10; Secondary 55E40.

Key words and phrases. Banach algebra, torsion, homotopy group.

${ }_{1}^{1}$ This research was supported by N.S.F. Grant GP-24182.

2 The author would like to thank Professor E. Faddell for his help. 
One checks that $\left(F^{*}\right)^{\sim}$ is the adjoint of $\tilde{F}$ and that $F \rightarrow \tilde{F}$ is a normpreserving isomorphism of $A(X, n)$ onto a $B^{*}$-subalgebra of $B(H)$.

The group of invertible elements of $A(X, n)$, which we denote by $G(X, n)$, is the set of all continuous functions from $X$ into $G L(n)$, the group of all invertible complex $n \times n$ matrices. Let $G_{e}(X, n)$ denote the component of the identity of $G(X, n)$. Then the elements of $G(X, n) / G_{e}(X, n)$ are just the homotopy classes of maps from $X$ into $G L(n)$ because two maps $f, g \in G(X, n)$ are homotopic if and only if $f g^{-1} \in G_{e}(X, n)$.

Notation. Let $S$ and $T$ be topological spaces. We let $[S, T]$ denote the space of homotopy classes of maps from $S$ into $T$ with the topology derived from the compact-open topology on the set $\operatorname{Map}(S, T)$ of continuous functions from $S$ into $T$. If $T$ is a topological group, then so is $\operatorname{Map}(S, T)$ under the pointwise multiplication of functions, and it is easily shown that $[S, T]$ is also a topological group $[4$, p. 34].

Proposition. Let $A(X, n), G(X, n), G_{e}(X, n)$ be defined as above. Then $G(X, n) / G_{e}(X, n)$ is homeomorphic and isomorphic to $[X, \mathscr{U}(n)]$ where $\mathscr{U}(n)$ denotes the group of all complex unitary $n \times n$ matrices.

Proof. We have $G(X, n) / G_{e}(X, n)=[X, G L(n)]$ because $G(X, n)$ is $\operatorname{Map}(X, G L(n))$ and two maps in $G(X, n)$ are homotopic if and only if they lie in the same component of $G(X, n)$. Now since $G L(n)$ is homeomorphic to $\mathscr{U}(n) \times \boldsymbol{R}^{n^{2}}\left[\mathbf{1}\right.$, p. 16] and $\boldsymbol{R}^{n^{2}}$ is contractible, every $f \in \operatorname{Map}(X$, $G L(n)$ is homotopic to a map $\tilde{f} \in \operatorname{Map}(X, \mathscr{U}(n))$. Thus $[X, G L(n)]$ is homeomorphic and isomorphic to $[X, \mathscr{U}(n)]$ under the map $f \rightarrow \tilde{f}$.

We now construct our examples by choosing appropriate spaces $X$.

EXAmple 1. Let $S^{k}$ denote the real $k$-sphere. Then the following statements hold for the algebra $A\left(S^{k}, n\right)$ :

(a) $\left[S^{k}, \mathscr{U}(n)\right] \cong \pi_{k}(\mathscr{U}(n))$,

(b) $G\left(S^{k}, 2\right) / G_{e}\left(S^{k}, 2\right) \cong \pi_{k}(\mathscr{U}(2)) \cong \pi_{k}\left(S^{3}\right)$.

In particular, $\pi_{k}\left(S^{3}\right)$ is finite and nontrivial if $4 \leqq k \leqq 22$.

Proof. Part (a) is true because both groups have the same elements, $S^{k}$ is a suspension and $\mathscr{U}(n)$ is an $H$-space (see [4, p. 44]). The first equality in part (b) follows from the Proposition. The second equality in (b) holds because $\mathscr{U}(2)$ is homeomorphic to $S^{3} \times S^{1}\left[5\right.$, p. 129] and so $\pi_{k}(\mathscr{U}(2))$ $=\pi_{k}\left(S^{3}\right) \oplus \pi_{k}\left(S^{1}\right)$. For $k>1, \pi_{k}\left(S^{1}\right)=0[5$, p. 111] and if $4 \leqq k \leqq 22$, then $\pi_{k}\left(S^{3}\right)$ is finite and nontrivial [6, pp. 186-188] and so for these $k$ at least $G\left(S^{k}, 2\right) / G_{e}\left(S^{k}, 2\right)$ is finite and nontrivial.

Note. The result may hold for other $k$ as well, but these are the only values of $\pi_{k}\left(S^{3}\right)$ given in the reference quoted above.

The algebras $A\left(S^{k}, n\right)$ always have abelian factor groups $G\left(S^{k}, n\right) / G_{e}\left(S^{k}, n\right)$ $\cong \pi_{k}(\mathscr{U}(n))$ because $\mathscr{U}(n)$ is an $H$-space [4, p. 44]. The following example of a complex Banach algebra with a nonabelian factor group $G / G_{e}$ is due to E. Fadell. 
EXAmple 2. Let $X=\mathscr{U}(2) \times \mathscr{U}(2)$. Then in the algebra $A(X, 2)$, $G(X, 2) / G_{e}(X, 2) \cong[X, \mathscr{U}(2)]$ is nonabelian.

PROOF. Define the following four maps on $\mathscr{U}(2) \times \mathscr{U}(2)$ :

$$
f(u, v)=u, \quad \varphi(u, v)=(v, u), \quad g(u, v)=v, \quad \mu(u, v)=u \cdot v
$$

where $(u, v) \in \mathscr{U}(2) \times \mathscr{U}(2)$ and the dot indicates multiplication in $\mathscr{U}(2)$. Then $[f]$ and $[g]$, the homotopy classes of maps from $X$ to $\mathscr{U}(2)$ which are homotopic to $f$ and $g$ respectively, are elements of $[X, \mathscr{U}(2)]$. Suppose $[X, \mathscr{U}(2)]$ is abelian. Then the maps $f \cdot g(u, v)=u \cdot v$ and $g \cdot f(u, v)=v \cdot u$ are homotopic. We denote this by $f \cdot g \sim g \cdot f$. Since $\mu(u, v)=u \cdot v$ $=f \cdot g(u, v)$ and $g \cdot f(u, v)=v \cdot u=\mu \circ \varphi(u, v)$, this is equivalent to saying $\mu \sim \mu \circ \varphi$. Let $\bar{\mu}$ and $\bar{\varphi}$ denote the restrictions of $\mu$ and $\varphi$, respectively, to $S \mathscr{U}(2) \times S \mathscr{U}(2)$. Then $\bar{\mu}: S \mathscr{U}(2) \times S \mathscr{U}(2) \rightarrow S \mathscr{U}(2)$ since $S \mathscr{U}(2)$ is a subgroup of $\mathscr{U}(2)$ and $\bar{\varphi}: S \mathscr{U}(2) \times S \mathscr{U}(2) \rightarrow S \mathscr{U}(2) \times S \mathscr{U}(2)$. Thus the above statements imply that $\bar{\mu} \sim \bar{\mu} \circ \bar{\varphi}$. Since $S \mathscr{U}(2)$ is homeomorphic to $S^{3}$, this is equivalent to saying that $\bar{\mu}$ is a homotopy commutative multiplication on $S^{3}$. This contradicts the fact that $S^{1}$ is the only sphere which admits a homotopy commutative multiplication $[2$, p. 192]. Hence $[f][g] \neq[g][f]$ and so $G(X, 2) / G_{e}(X, 2)$ is nonabelian.

If $B$ and $C$ are Banach algebras with groups $H$ and $K$ and identity components $H_{e}$ and $K_{e}$, respectively, one can form their direct sum $A=B \oplus C$ by adding and multiplying componentwise and letting

$$
\|(b, c)\|=\|b\|+\|c\| \quad \text { for } b \in B \text { and } c \in C .
$$

If $G$ is the group of invertible elements of $A$ and $G_{e}$ is its identity component then $G \cong H \oplus K$ and $G_{e} \cong H_{e} \oplus K_{e}$ and so $G / G_{e} \cong H / H_{e} \oplus K / K_{e}$. Thus, one can obtain more complicated groups $G / G_{e}$ by letting $A$ be, for example, the algebra of all continuous functions from the disjoint union of a sphere $S^{k}(4 \leqq k \leqq 22)$ and $\mathscr{U}(2) \times \mathscr{U}(2)$ into $M_{n}$. Then $A$ decomposes into the direct sum of algebras $A\left(S^{k}, n\right) \oplus A(\mathscr{U}(2) \times \mathscr{U}(2), n)$ and $G / G_{e}$ is the direct sum of the corresponding factor groups of these algebras. If we let $n=2$, this process yields an algebra whose factor group $G / G_{e}$ is nonabelian and has torsion.

\section{BIBLIOGRAPHY}

1. C. Chevalley, Theory of Lie groups. Vol. 1, Princeton Math. Series, vol. 8, Princeton Univ. Press, Princeton, N.J., 1946. MR 7, 412.

2. I. M. James, Multiplication on spheres. I, Proc. Amer. Math. Soc. 8 (1957), 192-196. MR 18, 752.

3. C. E. Rickart, General theory of Banach algebras, University Series in Higher Math., Van Nostrand, Princeton, N.J., 1960. MR 22 \# 5903.

4. E. H. Spanier, Algebraic topology, McGraw-Hill, New York, 1966. MR 35 \# 1007.

5. N. E. Steenrod, The topology of fibre bundles, Princeton Math. Series, vol. 14, Princeton Univ. Press, Princeton, N.J., 1951. MR 12, 522.

6. H. Toda, Composition methods in homotopy groups of spheres, Ann. of Math. Studies, no. 49, Princeton Univ. Press, Princeton, N.J., 1962. MR 26 \# 777.

Department of Mathematics, University of Wisconsin, Madison, Wisconsin 53706 